\title{
NDRG2 Expression Increases Apoptosis Induced by Doxorubicin in Malignant Breast Caner Cells
}

\author{
Myung-Jin KIM, Kyeongah KANG, Young YANG, and Jong-Seok LIM* \\ Department of Biological Science and the Research Center for Women's Diseases, \\ Sookmyung Women's University, Seoul 140-742, Republic of Korea
}

(Received July 7, 2009; Revised July 7, 2009; Accepted August 3, 2009)

\begin{abstract}
N-myc downstream-regulated gene 2 (NDRG2) has recently been found to be a tumor suppressor gene. Although it has been reported that NDRG2 expression in breast cancer cells decreases cell proliferation by inhibiting STAT3 activation via SOCS1 induction, the molecular mechanism of chemotherapeutic agent-induced apoptosis is not well known. To elucidate the effect of NDRG2 on the apoptotic pathway induced by doxorubicin, we established stable cell lines expressing NDRG2 and investigated the effect of NDRG2 expression on the doxorubicin-induced apoptosis. While STAT3 activation was remarkably inhibited by NDRG2 overexpression, the expression level of p21 was increased by NDRG2 expression. We confirmed that NDRG2-expressing cells treated with doxorubicin suppressed STAT3 activation and upregulated p21 expression. NDRG2 expression considerably enhanced TUNEL positive apoptotic cells, poly-ADP ribose polymerase (PARP) cleavage, release of cytochrome $c$ to cytosol, and caspase-3 activity in doxorubicin-induced apoptosis. Bid expression in a resting state and after treatment with doxorubicin increased in MDA-MB-231-NDRG2 cells compared to MDA-MB-231-mock cells. Meanwhile, Bcl-X $\mathrm{x}_{\mathrm{L}}$ expression decreased in MDA-MB-231-NDRG2 cells compared to MDA-MB-231-mock cells in a resting state and in doxorubicin-treated cells. Collectively, these data suggest that suppression of STAT3 activation by NDRG2 influences the sensitivity to doxorubicin-induced apoptosis of breast cancer cells and this may provide a potential therapeutic benefit to overcome the resistance against doxorubicin in breast cancer.
\end{abstract}

Keywords: N-myc downstream-regulated gene 2 (NDRG2), Doxorubicin, Breast cancer, Apoptosis

\section{INTRODUCTION}

Breast cancer is both the most common type of cancer and the most common cause of cancer-related death in women worldwide. Although there has been some improvement in survival with chemotherapy, serious side effects and the acquisition of multiple drug resistance are major obstacles in anti-cancer treatments. Making progress in our understanding of tumor resistance to chemotherapy and finding alternatives to current treatments are imperative.

Recent work has focused on multiple levels of various signaling pathways, including the signal transducer and activator of transcription 3 (STAT3) pathway, which plays an important role in cell proliferation, transformation, mor-

${ }^{*}$ Corresponding author

Tel: +82-2-710-9560 Fax: +82-2-2077-7322

E-mail: jslim@sookmyung.ac.kr tality, and survival (Wang et al., 2008). STAT3 is a member of the STAT family of latent, cytosolic transcription factors that directly relate signals from the plasma membrane to the nucleus. This protein is constitutively activated by aberrant upstream tyrosine kinase activity in a broad spectrum of human tumors (Costantino and Barlocco, 2008). Notably, constitutive activation of STAT3 has frequently been observed in breast cancer and malignant breast cancer cell lines. In addition, STAT3 activation in breast cancer cells decreases the efficiency of chemotherapy (Aggarwal et al., 2006; Gariboldi et al., 2007; Ishii et al., 2008), while STAT3 inhibition increases the proapoptotic effect of doxorubicin (Gariboldi et al., 2007). Thus, the identification of upstream genes regulating STAT3 activation is critical for increasing tumor cell sensitivity to anticancer drugs.

NDRG2 is a member of the N-myc downstream-regulated gene (NDRG) family, such as NDRG1-4, which is normally expressed in the brain, heart, and muscle (Kim et al., 
2008). Recently, it was reported that NDRG2 expression is down-regulated in a variety of carcinomas including liver cancer, pancreatic cancer (Hu et al., 2004), and meningioma (Lusis et al., 2005), and it is also associated with cell growth, differentiation, and apoptosis (Choi et al., 2007). In addition, NDRG2 expression in breast cancer cells has been shown to inhibit STAT3 activation via SOCS1 induction, followed by a decrease in cell proliferation (Park et al., 2007). Taken together, these data suggest that NDRG2 may be a new tumor suppressor gene candidate, and it is expected to contribute to targeting for cancer treatment.

Doxorubicin is a broad spectrum chemotherapeutic that exhibits activity against breast, lung, thyroid and ovary carcinomas, leukemia, and Hodgkin's and non-Hodgkin's lymphomas (Swift et al., 2008). In addition to its capacity to intercalate into DNA and to inhibit macromolecular biosynthesis, doxorubicin has been reported to interact directly with the cell membrane and to inhibit the enzyme topoisomerase II, where inhibition of DNA religation results in the induction of protein-associated strand breaks in DNA (Fornari et al., 1994).

In this study, we established NDRG2-overexpressing breast cancer cells in order to elucidate the effect of NDRG2 on the apoptotic pathway induced by doxorubicin. Phosphorylation of STAT3 was remarkably inhibited in MDA-MB-231-NDRG2 compared to MDA-MB-231-mock cells. In contrast, the level of p21 in MDA-MB-231-NDRG2 cells was significantly higher than that observed in MDA-MB-231-mock cells. We therefore examined the level of apoptosis after treatment with doxorubicin and we found that cytochrome $c$ release was markedly upregulated by NDRG2 overexpression, and that PARP cleavage and caspase-3 activation were more obvious in MDAMB-231-NDRG2 than in MDA-MB-231-mock cells. Expression of Bid was observed in MDA-MB-231-NDRG2 cells in a resting state, while after treatment with doxorubicin, the expression was significantly higher in MDA-MB-231NDRG2 cells than in MDA-MB-231-mock cells. On the contrary, $\mathrm{Bcl}-\mathrm{X}_{\mathrm{L}}$ expression was observed at a low level in MDA-MB-231-NDRG2 cells, compared to MDA-MB-231mock cells in a resting state, as well as under doxorubicin-treatment conditions. Thus, NDRG2 overexpression provides a potential therapeutic benefit for overcoming resistance to doxorubicin in breast cancer.

\section{MATERIALS AND METHODS}

\section{Chemicals and antibodies}

Doxorubicin (Dox) was purchased from Calbiochem (Merck Bioscience, San Diego, CA) and dissolved in ul- tra-pure water. The following antibodies were used: rabbit polyclonal anti-pSTAT3 (Tyr 705), anti-STAT3, anti-PARP (Cell Signaling Technology, Danvers, MA); rabbit anti-p21, rabbit anti-caspase-3, rabbit anti-cytochrome c, goat anti-Bid, rabbit anti-Bcl- $x_{\mathrm{L}}$, goat anti- $\beta$-actin (Santa Cruz, CA); and mouse anti-NDRG2 produced by our laboratory (Choi et al., 2007).

\section{Cell culture and treatment}

MDA-MB-231 cell lines and their cloned derivatives were grown in DMEM (Gibco/Invitrogen, Carlsbad, CA) supplemented with $10 \%$ heat-inactivated fetal bovine serum (FBS; Gibco/Invitrogen) and 1\% penicillin/streptomycin (Gibco/Invitrogen). Cells were maintained in an atmosphere of $5 \% \mathrm{CO}_{2}$ in a $37^{\circ} \mathrm{C}$ humidified incubator. The cells were treated with doxorubicin at various concentrations and times.

\section{Transfection of NDRG2 gene}

MDA-MB-231-NDRG2 clones were generated by stable transfection of MDA-MB-231 cells with the plasmid pCMVTaq2B containing the full length NDRG2 gene using Lipofectamine $^{\mathrm{TM}} 2000$ (Invitrogen, Carlsbad, CA). Briefly, MDA-MB-231 cells were plated at $70-80 \%$ confluency in media without antibiotics for $12 \mathrm{~h}$ and were transfected with $5 \mu \mathrm{g}$ of DNA per $1 \times 10^{6}$ cells the following day. Transfectants resistant to $1 \mathrm{mg} / \mathrm{ml}$ G418 (MP Biochemicals, Solon, $\mathrm{OH}$ ) were screened for NDRG2 expression. NDRG2 expression was confirmed by RT-PCR and Western blotting using an anti-NDRG2 antibody.

\section{RT-PCR}

Total RNA from harvested cells was isolated using TRIzol reagent (Invitrogen) and $5 \mu \mathrm{g}$ of total RNA was reverse transcribed (RT) to cDNA. For the RT reaction, RNA was first incubated with an oligo-(dT) primer at $70^{\circ} \mathrm{C}$ to denature the RNA secondary structure and then incubated at room temperature for $10 \mathrm{~min}$ to allow the primer to anneal to the RNA. Other components of RT, including dNTPs (Bioneer, Daejeon, Korea), M-MLV reverse transcriptase (Promega, Madison, WI), and RT buffer (Promega) were then added to the reaction. The $\mathrm{RT}$ reaction was incubated at $37^{\circ} \mathrm{C}$ for $1 \mathrm{~h}$ and then heated at $100^{\circ} \mathrm{C}$ for $5 \mathrm{~min}$. The RT reaction was then used as a template for a $20 \mu \mathrm{lPCR}$ reaction. PCR primers were purchased from GENOTECH or Bioneer (Daejeon, Korea). The following primer sequences were used. For actin: (sense) '5-CCA CAC CTT CTA CAA TGA GC-3', (antisense) 5'-TGA GGT AGT CAG TCA GGT CC-3'. For NDRG2: (sense) 5'-GGA TTC ATG GCG GAG CTG CAG GAG G-3' (antisense) 5'-GAA TTC 
TCA ACA GGA GAC CTC CAT GGT-3'. PCR products were electrophoresed on $1 \%$ agarose gels and visualized by ethidium bromide $(\mathrm{EtBr})$ staining.

\section{Western blot analysis}

For the preparation of whole cell lysates, cells were lysed on ice in Pro-prep ${ }^{\mathrm{TM}}$ reagent (iNtRON Biotechnology, Seongnam-Si, Gyeonggy-Do, Korea) for $30 \mathrm{~min}$. Supernatant fractions were recovered by $12,000 \mathrm{rpm}$ for $5 \mathrm{~min}$ at $4^{\circ} \mathrm{C}$. Isolation of the cytosolic fraction for cytochrome $\mathrm{c} \mathrm{de}$ tection was performed by quick lysis in digitonin. In brief, an equal volume of lysis buffer ( $9.4 \mu \mathrm{g}$ digitonin $/ 10^{6}$ cells; $500 \mathrm{mM}$ sucrose in PBS; $2 \mathrm{mM} \mathrm{NaH} \mathrm{PO}_{4}, 16 \mathrm{mM}$ $\mathrm{Na}_{2} \mathrm{HPO}_{4}, 150 \mathrm{mM} \mathrm{NaCl}, \mathrm{pH}$ 7.6) was added to a cell suspension of $5 \times 10^{6}$ cells in PBS. Cellular organelles were removed by centrifugation for $1 \mathrm{~min}$ at $12,000 \mathrm{rpm}$ and $4^{\circ} \mathrm{C}$. The supernatant cytosolic fraction was used for quantification of cytochrome $c$ by immunoblotting. Cell lysates containing equivalent amounts of total protein were mixed with SDS sample buffer and resolved on an SDS-polyacrylamide gel. Separated proteins were transferred to a PVDF membrane using a Bio-Rad transfer apparatus (Hercules, CA). Membranes were incubated in $4 \%$ BSA or $5 \%$ skim milk (BD Pharmingen, Heidelberg, Germany) in TBS-T (20 $\mathrm{mM}$ Tris-HCl, pH 7.5, $137 \mathrm{mM} \mathrm{NaCl}, 0.05 \%$ tween-20) for 1 $\mathrm{h}$ at room temperature, washed with TBS-T and then incubated with the primary antibody overnight at $4^{\circ} \mathrm{C}$. The membranes were washed with TBS-T and then antibody binding was followed by incubation with a peroxidase-conjugated goat anti-rabbit IgG, anti-mouse IgG secondary antibody (Jackson ImmunoResearch Laboratories, West Grove, PA) or bovine-anti-goat IgG HRP (Santa Cruz) at room temperature for $1 \mathrm{~h}$. Protein bands were visualized with a Chemiluminescent Substrate kit purchased from Pierce (Pierce, Rockford, IL) using a LAS 3000 imaging system (FUJIFILM Corporation, Tokyo, Japan).

\section{Trypan blue exclusion assay}

Cells $\left(4 \times 10^{5}\right.$ cells/well) were plated in $60 \mathrm{~mm}$ culture dishes. The cells were added to trypsin-EDTA (Gibco), harvested, and centrifuged at 1,200 rpm for $5 \mathrm{~min}$. Resuspended cells were mixed with $0.4 \%$ trypan blue and viable cells, which are visible using light microscopy, were enumerated using a hematocytometer.

\section{TUNEL assay}

The TUNEL assay was used to detect DNA fragmentation in apoptotic cells by direct end-labeling of cellular genomic DNA with a fluorescein-conjugated nucleotide, using the terminal deoxynucleotidyl transferase enzyme.
MDA-MB-231-wild type, -mock, and -NDRG2 cells $\left(1 \times 10^{5}\right.$ cells/well) were plated on poly-D-Lysine-coated coverslips in a 12-well plate. Cells were treated with doxorubicin for $24 \mathrm{~h}$ and then fixed with $4 \%$ formaldehyde for $1 \mathrm{~h}$ at $25^{\circ} \mathrm{C}$. Cells were then incubated with Blocking Solution $\left(3 \% \mathrm{H}_{2} \mathrm{O}_{2}\right.$ in absolute methanol) for $10 \mathrm{~min}$ at $25^{\circ} \mathrm{C}$. Following blocking, cells were permeabilized using a freshly prepared permeabilization solution ( $0.5 \%$ Triton X-100 in PBS) for $2 \mathrm{~min}$ on ice. The TUNEL reaction mixture, including both label solution and enzyme solution (Roche, Mannheim, Germany), was added to samples in the dark. After $1 \mathrm{~h}$ at $37^{\circ} \mathrm{C}$ in a humidified atmosphere in the dark, mounting solution with DAPI was added to samples and then samples were analyzed under a fluorescence microscope.

\section{Caspase activity assay}

Caspase activity was measured using the Ac-DEVDAFC caspase-3 fluorogenic substrate (Alexis, San Diego, CA). MDA-MB-231-wild type, -mock, and -NDRG2 cells were washed once with PBS, resuspended in lysis buffer (20 mM HEPES, pH 7.4, $100 \mathrm{mM} \mathrm{NaCl}, 0.5 \%$ NP-40, and $10 \mathrm{mM}$ DTT), and incubated on ice for $30 \mathrm{~min}$. After centrifugation $\left(12,000 \mathrm{rpm}, 20 \mathrm{~min}, 4^{\circ} \mathrm{C}\right)$, the supernatants were collected and immediately measured for protein concentration and caspase activity. Cell lysates $(100 \mu \mathrm{g})$ were mixed with $100 \mu \mathrm{M}$ of caspase-3 substrate. The mixture was incubated at $37^{\circ} \mathrm{C}$ for $1 \mathrm{~h}$ and monitored with a spectrophotometer at excitation and emission wavelengths of $400 \mathrm{~nm}$ and $505 \mathrm{~nm}$, respectively.

\section{Statistical analysis}

Results are presented as mean \pm standard deviation (S.D.). All experiments were repeated at least three times, with documented reproducibility. Data were analyzed for statistical significance using the Student's $t$-test. $p$-values of less than 0.05 were considered statistically significant.

\section{RESULTS}

\section{NDRG2 overexpression inhibits STAT3 activation in MDA-MB-231 cells}

To examine the role of NDRG2 in breast cancer cells, MDA-MB-231 wild type cells were transfected with the NDRG2 gene. First, selected MDA-MB-231-NDRG2 transfectants were assayed for NDRG2 mRNA and protein expression using RT-PCR and Western blotting, respectively. As shown in Fig. 1A, MDA-MB-231-wild type and -mock transfected cells expressed NDRG2 mRNA and protein at a low level, whereas NDRG2-transfected MDA-MB-231 cells displayed an intense expression of 


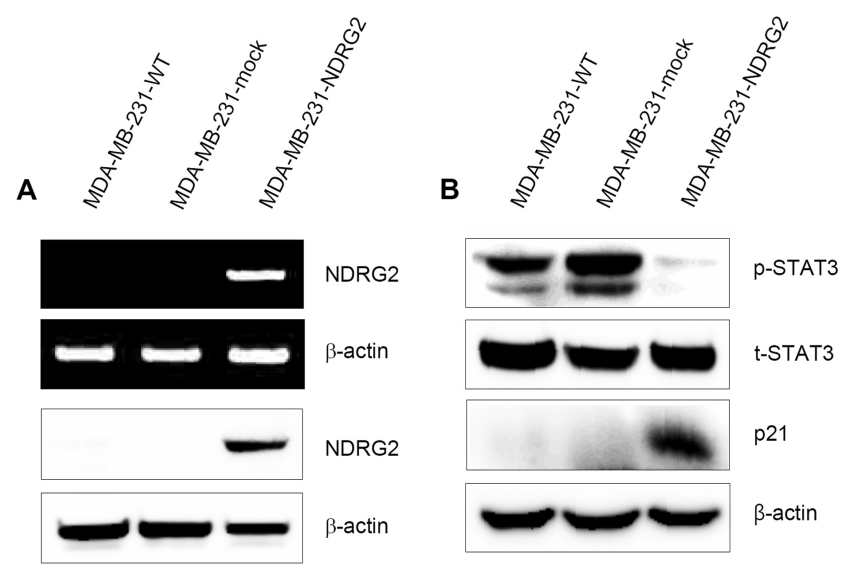

Fig. 1. Expression levels of NDRG2 and p-STAT3 in MDA-MB-231-wild, -mock, and -NDRG2 cells. (A) MDA-MB-231 cells stably transfected with $\mathrm{pCMV}-\mathrm{Taq} 2 \mathrm{~B} /$ human NDRG2 vector were maintained in DMEM supplemented with $10 \%$ FBS containing $1 \mathrm{mg} / \mathrm{ml} \mathrm{G} 418$. Expression levels of NDRG2 mRNA and protein in MDA-MB-231-wild, -mock, and -NDRG2 cells were confirmed by RT-PCR (upper panel) and Western blot analysis (lower panel), respectively. (B) The expression level of STAT3 phosphorylation and p21 was examined by Western blot analysis.

NDRG2 mRNA and protein. In addition, phosphorylation of STAT3 (Tyr 705) in relation to cell proliferation was remarkably inhibited in MDA-MB-231-NDRG2 cells compared to MDA-MB-231-mock cells, and the expression level of p21 increased in MDA-MB-231-NDRG2 cells (Fig. $1 \mathrm{~B}$ ), as demonstrated in a previous study (Park et al., 2007).

\section{NDRG2 overexpression increases cell growth retardation induced by doxorubicin}

According to a report that STAT3 inhibition increases the proapoptotic effect of doxorubicin (Gariboldi et al., 2007), we examined the effect of NDRG2 on apoptosis induced by doxorubicin. First, MDA-MB-231-wild type, -mock, and -NDRG2 cells were treated with various concentrations (0.125-5 $\mu \mathrm{M})$ of doxorubicin for $72 \mathrm{~h}$, after which the MTT assay was performed to examine cell viability (data not shown). After treatment with $1 \mu \mathrm{M}$ of doxorubicin, MDA-MB-231 wild type cells showed an approximate $50 \%$ reduction in cell viability, compared to untreated controls. From these results, $1 \mu \mathrm{M}$ doxorubicin was determined to be sufficient to induce apoptosis. These data were confirmed by trypan blue exclusion studies (Fig. $2 A)$. Cells $\left(4 \times 10^{5}\right)$ were plated in $60 \mathrm{~mm}$ dishes and were then treated with different concentrations $(0.5-10 \mu \mathrm{M})$ of doxorubicin for $24 \mathrm{~h}$. A previous study showed that MDA-MB-231 cells expressing NDRG2 had a reduced

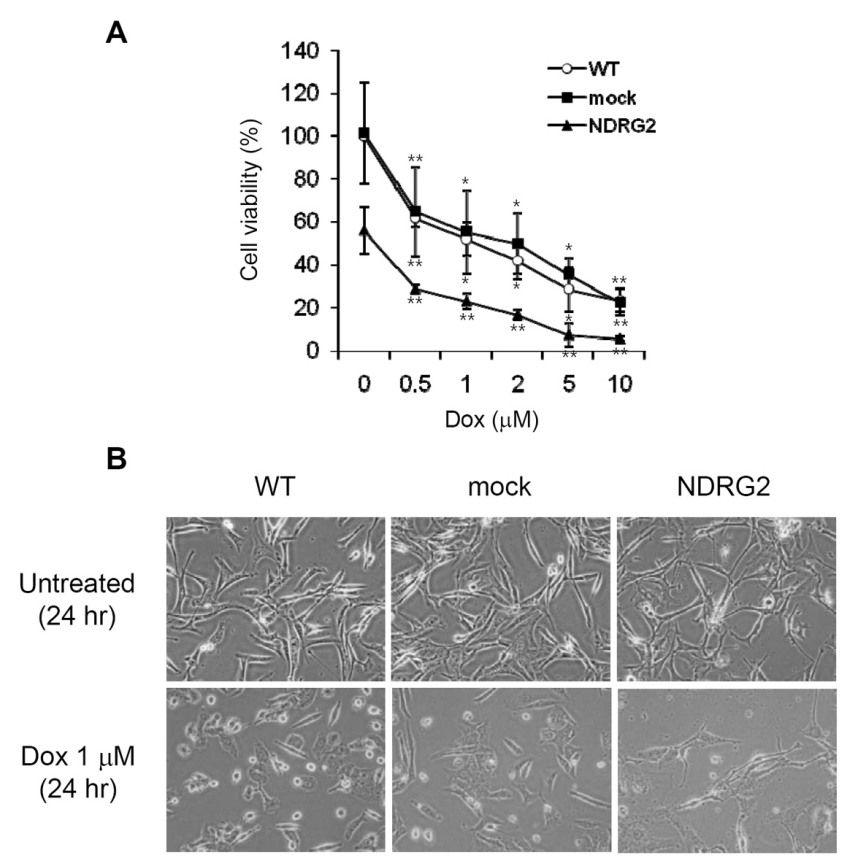

Fig. 2. Changes of cell viability and morphology in MDA-MB231-wild type (WT), -mock, and -NDRG2 cells by doxorubicin treatment. (A) Cells $\left(4 \times 10^{5}\right)$ were plated in $60 \mathrm{~mm}$ dishes and were then treated with different concentrations $(0.5-10 \mu \mathrm{M})$ of doxorubicin (Dox). After $24 \mathrm{~h}$, cells were stained with trypan blue and cell numbers were counted with a hematocytometer. Data represent the mean \pm S.D. of three independent experiments. ${ }^{*} p<0.05,{ }^{* *} p<0.01$ control versus treatment group. (B) Cell morphology following $24 \mathrm{~h}$ exposure of doxorubicin was observed under a phase contrast microscope.

growth rate compared to wild or mock control cells (Park et al., 2007). Likewise, MDA-MB-231-NDRG2 cells showed 40 percent lower cell viability than MDA-MB-231-wild type and -mock cells in a resting state. Doxorubicin-treatment decreased cell viability in a dose-dependent manner. A significant inhibition was observed in particular in NDRG2overexpressing cells. After treatment with $1 \mu \mathrm{M}$ doxorubicin, MDA-MB-231-WT and -mock cells showed a cell viability of 60 percent of the untreated group, while MDAMB-231-NDRG2 cells had a cell viability of 30 percent of the untreated group. As shown in Fig. 2B, changes in cell morphology and density after a $24 \mathrm{~h}$ treatment with doxorubicin were also observed. One unique morphological change appeared as a flattening and unclear edge of the cells. These features were particularly remarkable in NDRG2overexpressing cells. These results suggest the possibility that NDRG2 expression may affect cytotoxic changes in cancer cells following treatment with doxorubicin. 
A
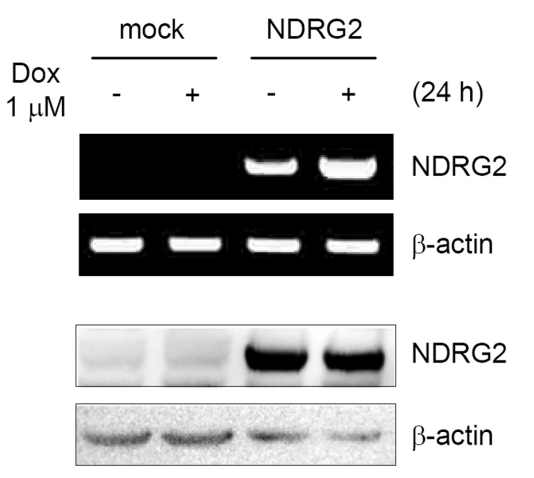

B

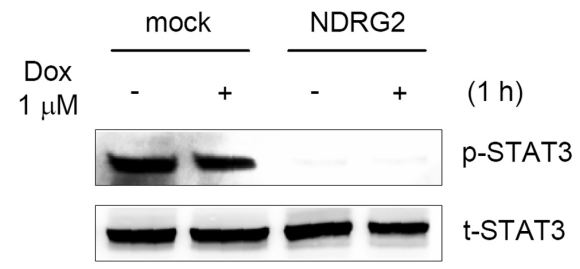

C

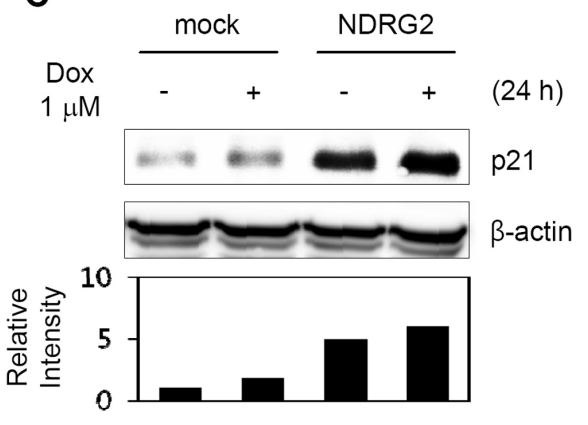

Fig. 3. The expression levels of NDRG2 mRNA and protein following treatment with doxorubicin and the effect of NDRG2 overexpression on phospho-STAT3 and p21 expression. (A) Expression levels of NDRG2 mRNA and protein of MDA-MB-231-mock and -NDRG2 cells following treatment with doxorubicin $(1 \mu \mathrm{M})$ for $24 \mathrm{~h}$. (B) Expression level of phospho-STAT3 protein in doxorubicin-treated cells for the indicated time. (C) Expression level of p21 was also examined by Western blotting, and relative band intensity was quantified.

\section{Enhancement of sensitivity to doxorubicin-induced cell death by NDRG2 overexpression}

The expression levels of NDRG2 mRNA and protein after treatment with doxorubicin were examined and are shown in Fig. 3A. NDRG2 mRNA expression was slightly increased in doxorubicin-treated MDA-MB-231-NDRG2 cells, whereas it was not detected in mock control cells, even after doxorubicin treatment. The expression levels of phospho-STAT3 and p21 protein were also examined after treatment with doxorubicin (Fig. 3B, C). In MDA-MB-231 wild type cells treated with doxorubicin, p21 expression was slightly increased compared to that in mock control cells. Suppression of STAT3 activation by NDRG2 was sustained under treatment with doxorubicin, and p21 expression was higher in MDA-MB-231-NDRG2 cells than control cells, following doxorubicin treatment. These results suggest that inhibition of STAT3 activation by NDRG2 may enhance the proapoptotic effect of doxorubicin.

\section{Doxorubicin-induced apoptosis is increased by NDRG2 overexpression}

To examine the effect of NDRG2 gene expression on apoptosis induced by doxorubicin, the TUNEL assay was performed. As shown in Fig. 4, TUNEL-positive apoptotic cells were markedly increased in MDA-MB-231-NDRG2 cells after treatment with doxorubicin, when compared to mock controls. These data suggest that NDRG2 expression may enhance the induction of apoptosis.

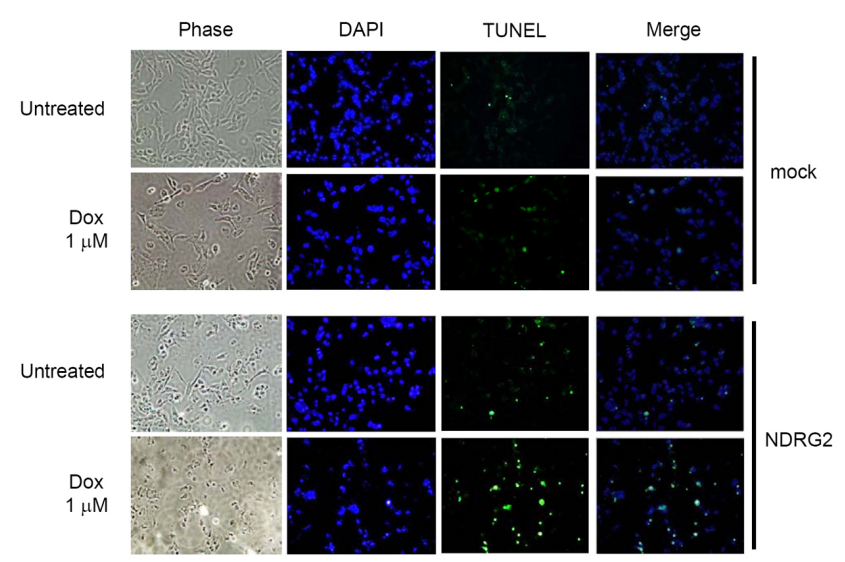

Fig. 4. Effect of NDRG2 overexpression on doxorubicin-induced apoptosis. After $24 \mathrm{~h}$ treatment with $1 \mu \mathrm{M}$ doxorubicin, TUNEL assay for apoptotic cells was performed and the results are shown as a fluorescence microscopic image (magnification, $\times 200$ ). The data shown are representative images of TUNEL staining of MDA-MB-231-mock and -NDRG2 cells of three independent experiments.

\section{Expression pattern of apoptotic proteins in MDA-MB- 231-NDRG2 cells after treatment with doxorubicin}

To investigate whether NDRG2 overexpression affects the expression of apoptosis-related molecules, we examined the expression levels of cytochrome $\mathrm{c}$, Bid, Bcl- $\mathrm{x}$, and PARP proteins by performing Western blot analysis (Fig. 5). Cytochrome c release was upregulated by NDRG2 overexpression, which was more pronounced in doxorubicin-treated cells. Moreover, expression of Bid in MDA- MB-231-NDRG2 cells was significantly higher after 


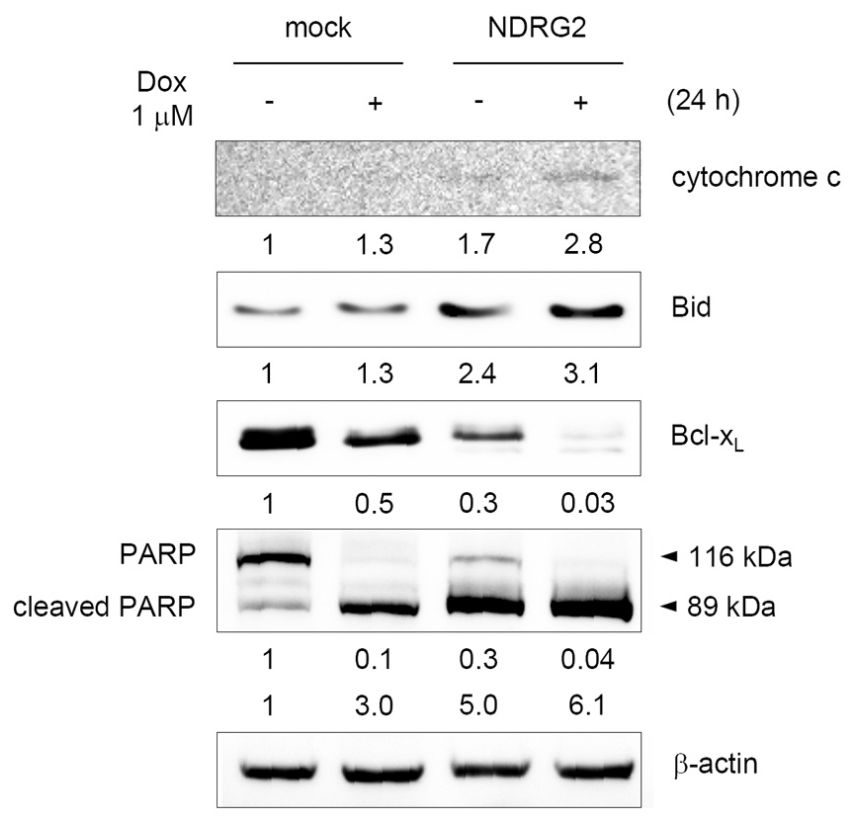

Fig. 5. Effect of NDRG2 overexpression on the expression of cytochrome c, Bid, Bcl- $\mathrm{x}_{\mathrm{L}}$, and PARP protein. After treatment with $1 \mu \mathrm{M}$ doxorubicin for $24 \mathrm{~h}$, cytochrome $\mathrm{c}$ was detected by Western blotting following the isolation of the cytosolic fraction by using lysis buffer with digitonin $\left(9.4 \mu \mathrm{g} / 10^{6}\right.$ cells). Expression levels of Bid and Bcl- $\mathrm{X}_{\mathrm{L}}$ (pro- and anti-apoptotic Bcl-2 family, respectively) proteins and PARP cleavage in MDA-MB-231mock and -NDRG2 cells were determined by Western blotting and then relative band density was quantified.

treatment with doxorubicin than that in mock controls. In contrast, a low level of $\mathrm{Bcl}-\mathrm{x}_{\mathrm{L}}$ expression was observed in MDA-MB-231-NDRG2 cells compared to MDA-MB-231mock cells in a resting state as well as following doxorubicin treatment. In addition, the cleaved form of PARP (89 $\mathrm{kDa}$ ) showed a time-dependent increase in MDA-MB-231mock cells after treatment with $1 \mu \mathrm{M}$ doxorubicin (data not shown). Although the treatment with $1 \mu \mathrm{M}$ doxorubicin or NDRG2 overexpression increased PARP cleavage, it was strongest in MDA-MB-231-NDRG2 cells. These results indicate that sensitivity to doxorubicin-induced apoptosis in breast cancer cell lines can be increased by NDRG2 overexpression.

\section{NDRG2 overexpression enhances caspase-3 activity induced by treatment with doxorubicin}

Caspase-3 functions as a key regulatory caspase through the cleavage of numerous cell death substrates, leading to cellular dysfunction and destruction. Thus, the expression level of active caspase- 3 protein following treatment with doxorubicin for $36 \mathrm{~h}$ was examined to confirm the apoptotic effect of doxorubicin in NDRG2-overexpress-
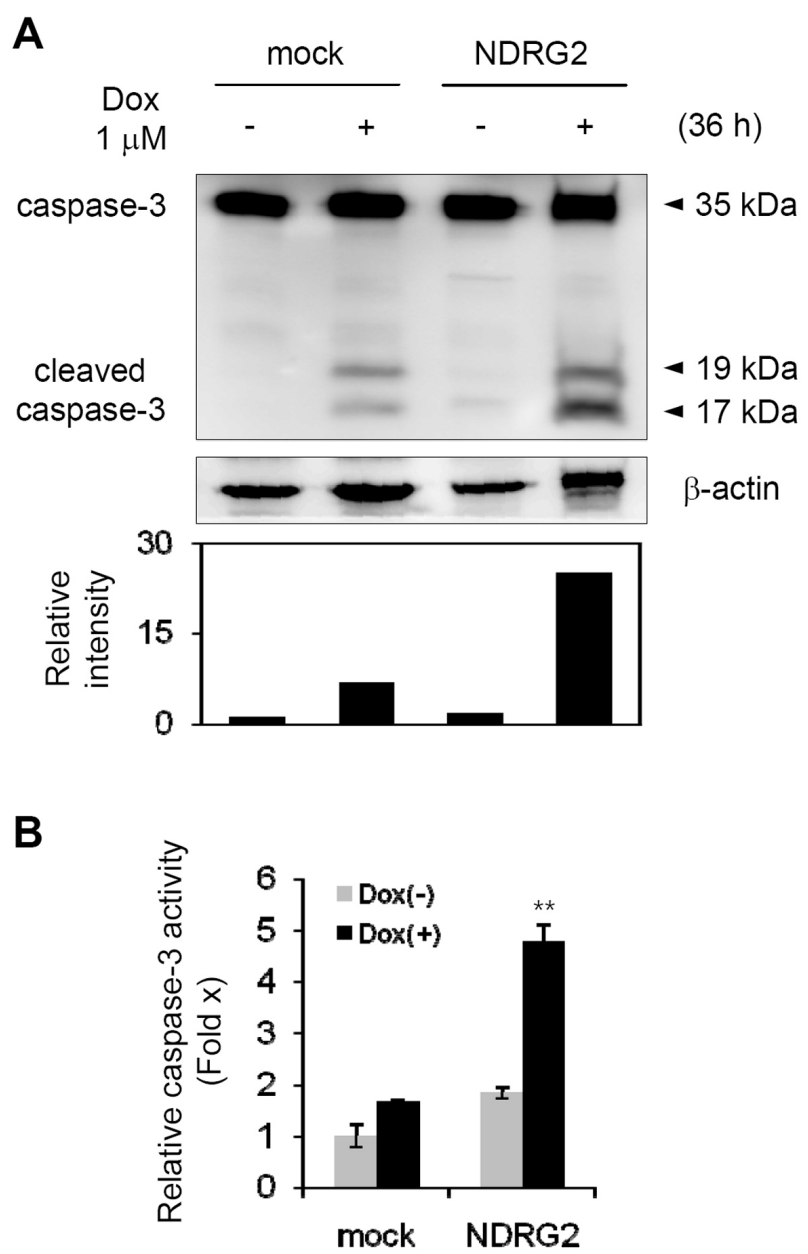

Fig. 6. Increase of caspase-3 activity by NDRG2 overexpression. (A) After exposure to $1 \mu \mathrm{M}$ doxorubicin for $36 \mathrm{~h}$, MDA-MB-231-mock and -NDRG2 cells were harvested and then the expression level of cleaved caspase-3 protein was detected by Western blotting, and relative band density of cleaved caspase-3 (17 kDa) was quantified. (B) In addition to the caspase- 3 protein level by Western blotting, caspase-3 activity assay using Ac-DEVD-AFC as a caspase-3-specific substrate was conducted in the presence of $1 \mu \mathrm{M}$ doxorubicin for $24 \mathrm{~h}$. Data are presented as the mean \pm S.D. from three similar experiments. ${ }^{* *} p<0.01$ MDA-MB-231-NDRG2 versus MDA-MB-231-mock cells.

ing cells (Fig. 6A). A significant induction of caspase-3 cleavage after treatment with doxorubicin was observed in MDA-MB-231-NDRG2 cells compared to mock control cells. Additionally, to confirm caspase-3 activation by doxorubicin treatment, the caspase-3 activity was measured using AC-DEVD-AFC as a caspase-3 substrate (Fig. 6B). Similar to the expression level of cleaved caspase-3 protein, caspase-3 activity was dramatically increased in MDA-MB-231-NDRG2 cells compared to MDA-MB-231- 
mock cells, especially after doxorubicin treatment. Collectively, these data suggest that NDRG2 overexpression may influence sensitivity to doxorubicin-induced apoptosis of breast cancer cells by inducing caspase- 3 activation.

\section{DISCUSSION}

The NDRG family consists of four members, NDRG1, NDRG2, NDRG3, and NDRG4, which share roughly $60 \%$ identity in their amino acid residues (Okuda and Kondoh, 1999; Zhou et al., 2001; Lachat et al., 2002; Qu et al., 2002). The members of this family have different tissue expression patterns, indicating that they may play distinct roles, but some exhibit similarities in either expression regulation or biological functions. Importantly, human NDRG2 was proposed to be a candidate tumor suppressor gene, due to its reduced expression in many cancer tissues including meningioma (Lusis et al., 2005), lung cancer, pancreatic cancer, and liver cancer (Hu et al., 2004), as well as its ability to inhibit proliferation in certain cancer cells like glioblastoma (Deng et al., 2003). Recently, it has been shown that NDRG2 can regulate cytokine signaling in breast cancer cells through the regulation of SOCS1 expression, which is capable of modulating STAT3 signaling (Park et al., 2007).

Compared with normal cells and tissues, constitutively activated STATs have been detected in a wide variety of human cancer cell lines and primary tumors. STATs are activated by tyrosine phosphorylation, which is normally a transient and tightly regulated process. In tumor cells, constitutive activation of STATs is linked to the persistent activity of tyrosine kinases. Numerous studies have demonstrated constitutive activation of STATs, especially STAT3, in a large number of diverse human tumor cell lines. Inhibition of constitutive STAT3 activation in diverse tumor cell lines, by the blocking of tyrosine kinase signaling using small-molecule inhibitors, has been repeatedly associated with growth suppression and induction of cell death (Epling-Burnette et al., 2001; Garcia et al., 2001; Aggarwal et al., 2006). In addition, it has been reported that upregulation of STAT3 phosphorylation in breast cancer cells reduces the efficiency of chemotherapy (Aggarwal et al., 2006; Gariboldi et al., 2007; Ishii et al., 2008), whereas suppression of STAT3 activation increases the proapoptotic effect of doxorubicin (Gariboldi et al., 2007). Accordingly, we assumed here that inhibition of STAT3 activation by NDRG2 in breast cancer cells may promote apoptosis induced by a chemotherapeutic agent, such as doxorubicin.

To support this hypothesis, NDRG2-overexpressing transfectants were established using MDA-MB-231 wild type cells. Overexpression of NDRG2 in MDA-MB-231 cells has been shown to inhibit cell proliferation (Park et al., 2007). The expression level of $p 21$ protein related to cell cycle arrest was conspicuously increased in MDA-MB231-NDRG2 cells, compared to mock control cells. Additionally, NDRG2 overexpression significantly inhibited the phosphorylation of STAT3 (Tyr 705). In order to elucidate the effect of NDRG2 on the apoptotic pathway induced by a chemotherapeutic agent and the extent of sensitivity to an anticancer drug, doxorubicin, a widely used chemotherapeutic agent, was utilized to induce apoptosis in MDA-MB-231-wild type, -mock, and -NDRG2 cells. Our results demonstrated that MDA-MB-231-NDRG2 cells show a significant reduction in cell viability compared to wild or mock control cells in the resting state and after exposure to the indicated concentrations of doxorubicin. By TUNEL assay, NDRG2-overexpressing cells treated with doxorubicin displayed a perceptible increase in TUNEL-positive apoptotic cells compared to MDA-MB-231-mock cells. The expression levels of proteins related to apoptosis, especially those involved in the mitochondrial pathway, were detected by Western blot analysis. Above all, MDA-MB-231NDRG2 cells displayed upregulated cytochrome $c$ release from the mitochondria into the cytosol after exposure to doxorubicin. In addition, since the mitochondrial pathway is regulated by activation of the Bcl-2 family, including both pro- (Bid, Bad, Bax, and Bim) and anti-apoptotic proteins (Bcl- $\mathrm{X}_{\mathrm{L}}$ and Bcl-2) (Johnstone et al., 2002), some Bcl-2 family proteins were also investigated by immunoblotting. Expression of Bid in NDRG2 transfectants was more increased than in mock controls following treatment with doxorubicin. On the contrary, Bcl-XL expression was downregulated in NDRG2-transfected cells compared to mocktransfected cells both prior to and following doxorubicin treatments. Accordingly, these data suggest that NDRG2 overexpression leads to more sensitive apoptotic effects in the doxorubicin-treated condition. Next, the extent of PARP cleavage was determined after various times and conditions of doxorubicin treatment. Originally, PARP detects and signals DNA strand breaks generated either directly during base excision repair, or indirectly by genotoxic agents. PARP is activated at an intermediate stage of apoptosis and is then cleaved and inactivated at a late stage by caspase- 3 and -7 (Decker and Muller, 2002). The expression level of cleaved PARP increased in MDA-MB231-NDRG2 cells treated with doxorubicin, compared to that in mock control cells. Finally, activation of caspase-3, involved in the final steps of the intrinsic apoptotic pathway (Ghobrial et al., 2005), was evaluated by Western blot 
analysis and caspase- 3 activity assay. Caspase- 3 protein levels were significantly increased in NDRG2-overexpressing cells compared to mock controls following treatment with doxorubicin. Furthermore, caspase-3 activity was upregulated approximately 5-fold in MDA-MB-231-NDRG2 cells after exposure to doxorubicin, significantly higher than in mock controls. Activity of caspase-8 or 9 following treatment with doxorubicin was not increased in MDA-MB231-NDRG2 cells (data not shown), indicating the specific effect of NDRG2 overexpression on caspase activity at a later stage. Finally, based on a recent study demonstrating that NDRG2 is involved in p53-mediated apoptosis (Lui et al., 2008), we tested whether NDRG2 overexpression affects p53 expression in MDA-MB-231 cells. As described in the same paper, it is known that MDA-MB-231 cells have mutant $\mathrm{p} 53$, and we therefore did not detect a change of p53 expression in MDA-MB-231-NDRG2 cells following treatment with doxorubicin (data not shown), suggesting that cell death of MDA-MB-231-NDRG2 cells induced by doxorubicin may not be dependent on p53 activity.

Taken together, our findings suggest that the effects of NDRG2 on the apoptotic pathway activated by doxorubicin may help enhance the effectiveness of chemotherapy. Suppression of STAT3 activation by NDRG2 influences sensitivity to doxorubicin-induced apoptosis of breast cancer cells, and these results may confer a potential therapeutic benefit to overcome the resistance against doxorubicin in breast cancer.

\section{ACKNOWLEDGMENTS}

This work was financially supported by the Sookmyung Women's University Research Grants 2009.

\section{REFERENCES}

Aggarwal, B. B., Sethi, G., Ahn, K. S., Sandur, S. K., Pandey, M. K., Kunnumakkara, A. B., Sung, B. and Ichikawa, H. (2006). Targeting signal-transducer-and-activator-of-transcription-3 for prevention and therapy of cancer: modern target but ancient solution. Ann. N. Y. Acad. Sci. 1091, 151-169.

Choi, S. C., Yoon, S. R., Park, Y. P., Song, E. Y., Kim, J. W., Kim, W. H., Yang, Y., Lim, J. S. and Lee, H. G. (2007). Expression of NDRG2 is related to tumor progression and survival of gastric cancer patients through Fas-mediated cell death. Exp. Mol. Med. 39, 705-714.

Costantino, L. and Barlocco, D. (2008). STAT 3 as a target for cancer drug discovery. Curr. Med. Chem. 15, 834-843.

Decker, P. and Muller, S. (2002). Modulating poly (ADP-ribose) polymerase activity: potential for the prevention and therapy of pathogenic situations involving DNA damage and oxidative stress. Curr. Pharm. Biotech. 3, 275-283.

Deng, Y., Yao, L., Chau, L., Ng, S. S., Peng, Y., Liu, X., Au, W.
S., Wang, J., Li, F., Ji, S., Han, H., Nie, X., Li, Q., Kung, H. F., Leung, S. Y. and Lin, M. C. (2003). N-Myc downstreamregulated gene 2 (NDRG2) inhibits glioblastoma cell proliferation. Int. J. Cancer 106, 342-347.

Epling-Burnette, P. K., Liu, J. H., Catlett-Falcone, R., Turkson, J., Oshiro, M., Kothapalli, R., Li, Y., Wang, J. M., Yang-Yen, H. F., Karras, J., Jove, R. and Loughran, T. P. Jr. (2001). Inhibition of STAT3 signaling leads to apoptosis of leukemic large granular lymphocytes and decreased Mcl-1 expression. J. Clin. Invest. 107, 351- 362.

Fornari, F. A., Randolph, J. K., Yalowich, J. C., Ritke, M. K. and Gewirtz, D. A. (1994). Interference by doxorubicin with DNA unwinding in MCF-7 breast tumor cells. Mol. Pharmacol. 45, 649-656.

Garcia, R., Bowman, T. L., Niu, G., Yu, H., Minton, S., MuroCacho, C. A., Cox, C. E., Falcone, R., Fairclough, R., Parsons, S., Laudano, A., Gazit, A., Levitzki, A., Kraker, A. and Jove, R. (2001). Constitutive activation of Stat3 by the Src and JAK tyrosine kinases participates in growth regulation of human breast carcinoma cells. Oncogene 20, 2499-2513.

Gariboldi, M. B., Ravizza, R., Molteni, R., Osella, D., Gabano, E. and Monti, E. (2007). Inhibition of Stat3 increases doxorubicin sensitivity in a human metastatic breast cancer cell line. Cancer Lett. 258, 181-188.

Ghobrial, I. M., Witzig, T. E. and Adjei, A. A. (2005). Targeting apoptosis pathways in cancer therapy. CA Cancer J. Clin. 55, 178-194.

Hu, X. L., Liu, X. P., Lin, S. X., Deng, Y. C., Liu, N., Li, X. and Yao, L. B. (2004). NDRG2 expression and mutation in human liver and pancreatic cancers. World J. Gastroenterol. 10, 3518-3521.

Ishii, Y., Waxman, S. and Germain, D. (2008). Tamoxifen stimulates the growth of cyclin D1-overexpressing breast cancer cells by promoting the activation of signal transducer and activator of transcription 3. Cancer Res. 68, 852-860.

Johnstone, R. W., Ruefli, A. A. and Lowe, S. W. (2002). Apoptosis: a link between cancer genetics and chemotherapy. Cell 108, 153-164.

Kim, Y. J., Yoon, S. Y., Kim, J. T., Choi, S. C., Lim, J. S., Kim, J. H., Song, E. Y., Lee, H. G., Choi, I. and Kim, J. W. (2008). NDRG2 suppresses cell proliferation through down-regulation of AP-1 activity in human colon carcinoma cells. Int. J. Cancer 124, 7-15.

Lachat, P., Shaw, P., Gebhard, S., van Belzen, N., Chaubert, P. and Bosman, F. T. (2002). Expression of NDRG1, a differentiation-related gene, in human tissues. Histochem. Cell Biol. 118, 399-408.

Lui, N., Wang, L., Li, X., Yang, Q., Liu, X., Zhang, J., Zhang, J., Wu, Y., Ji, S., Zhang, Y., Yang, A., Han, H. and Yao, L. (2008). N-Myc downstream-regulated gene 2 is involved in p53-mediated apoptosis. Nucl. Acid Res. 36, 5335-5349.

Lusis, E. A., Watson, M. A., Chicoine, M. R., Lyman, M., Roerig, P., Reifenberger, G., Gutmann, D. H. and Perry, A. (2005). Integrative genomic analysis identifies NDRG2 as a candidate tumor suppressor gene frequently inactivated in clinically aggressive meningioma. Cancer Res. 65, 71217126.

Okuda, T. and Kondoh, H. (1999). Identification of new genes ndr2 and ndr3 which are related to Ndr1/RTP/Drg1 but show distinct tissue specificity and response to N-myc. Biochem. 
Biophys. Res. Commun. 266, 208-215.

Park, Y., Shon, S. K., Kim, A., Kim, K. I., Yang, Y., Cho, D. H., Lee, M. S. and Lim, J. S. (2007). SOCS1 induced by NDRG2 expression negatively regulates STAT3 activation in breast cancer cells. Biochem. Biophys. Res. Commun. 363, 361367.

Qu, X., Zhai, Y., Wei, H., Zhang, C., Xing, G., Yu, Y. and He, F. (2002). Characterization and expression of three novel differentiation-related genes belong to the human NDRG gene family. Mol. Cell. Biochem. 229, 35-44.

Swift, L. P., Cutts, S. M., Nudelman, A., Levovich, I., Rephaeli, A. and Phillips, D. R. (2008). The cardio-protecting agent and topoisomerase II catalytic inhibitor sobuzoxane enhances
doxorubicin-DNA adduct mediated cytotoxicity. Cancer Chemother. Pharmacol. 61, 739-749.

Wang, Y. A., Johnson, S. K., Brown, B. L., McCarragher, L. M., Al-Sakkaf, K., Royds, J. A. and Dobson, P. R. (2008). Enhanced anti-cancer effect of a phosphatidylinositol-3 kinase inhibitor and doxorubicin on human breast epithelial cell lines with different p53 and oestrogen receptor status. Int. J. Cancer 123, 1536-1544.

Zhou, R. H., Kokame, K., Tsukamoto, Y., Yutani, C., Kato, H. and Miyata, T. (2001). Characterization of the human NDRG gene family: a newly identified member, NDRG4, is specifically expressed in brain and heart. Genomics 73, 86-97. 